\title{
A MODIFIED PULSAR MODEL Green Function period distribution
}

\author{
L.F. Chen, S.R. Liang \\ Department of Physics \\ Beijing Normal University \\ Beijing, China
}

The downward accelerated $\mathrm{e}^{-}$in the "unfavorable " zone, like that in the "favorable" one, will emit $\gamma$-photons, which in turn convert into $e^{ \pm}$ pairs in some places near the surface of stars. But what happens, which is different from that in the "favorable " zone, is that some $\gamma$-photons will travel through a long distance before their conversion. This makes it possible that some $\gamma$-photons arrive at the "diode " district in the "favorable" zone. The magnetic conversion of pairs is much easier to happen than that occured in the "favorable" zone, where the $\gamma$-photons are created by the primary $e^{-}$beam. The existence of dense $e^{ \pm}$plasma near the

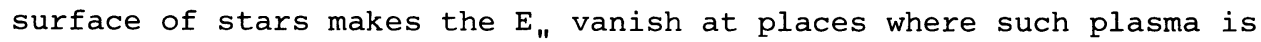
present.

In this paper, we have introduced $R^{\prime}$, which is the radius of the surface of dense $e^{ \pm}$plasma due to photon ${ }^{*}$ pair-production where $E_{"}$ is shorted out. $R_{*}^{\prime}$ will get large drastically when the pulsar period drops below a certain value.

Some new results have been obtained.

When $R_{*}^{\prime}$ becomes large the electron acceleration mechanism looses its efficiency and there would be a substantial reduction in radiation emitted from pulsars. This mechanism provides an explanation for the observed distribution of pulsar periods.

When the angle between the magnetic dipole axis and the rotation axis approaches $90^{\circ}$, potential $|\Phi|$ increases and the outflowing particles in the "unfavorable " zone can produce $\gamma$-ray which can create dense $e^{ \pm}$ plasma like the behavior of electrons in the "favorable" zone. The pulse we receive will come from both poles of the pulsars.

The energy of the outflowing positrons from the "unfavorable " zone can reach $1000 \mathrm{Gev}$ ( if radiation reaction is neglected). Such high energy positrons have been observed in interstellar cosmic rays. 